\title{
Lymphatic Mapping and Sentinel Lymph Node Biopsy for Patients With Local Recurrence After Breast-Conservation Therapy
}

\author{
Erika A. Newman, MD, ${ }^{1}$ Vincent M. Cimmino, MD,${ }^{1}$ Michael S. Sabel, MD, ${ }^{1}$ \\ Kathleen M. Diehl, MD, ${ }^{1}$ Kirk A. Frey, MD ${ }^{2}$ Alfred E. Chang, MD, ${ }^{1}$ \\ and Lisa A. Newman, MD, MPH ${ }^{1}$ \\ ${ }^{1}$ Department of Surgery, Comprehensive Cancer Center, University of Michigan, 1500 East Medical Center Drive, Ann Arbor, \\ Michigan 48109 \\ ${ }^{2}$ Department of Radiology/Nuclear Medicine, Comprehensive Cancer Center, University of Michigan, 1500 East Medical Center \\ Drive, Ann Arbor, Michigan 48109
}

Breast cancer incidence rates continue to increase among American women; however, earlier detection of disease with screening mammography, coupled with advances in breast cancer treatment, is resulting in overall outcome improvements and decreased

Received December 7, 2004; accepted August 16, 2005; published online January 1, 2006.

Address correspondence and reprint requests to: Lisa A. Newman, MD, MPH; E-mail: lanewman@umich.edu

Published by Springer Science+Business Media, Inc. @ 2006 The Society of Surgical Oncology, Inc. mortality rates. ${ }^{1}$ These trends will result in larger numbers of patients treated by breast-conservation therapy (consisting of lumpectomy and adjuvant breast radiotherapy), and the preserved breast is an ongoing source for additional neoplastic events in the form of true local recurrence or new primary tumors. Salvage mastectomy is the standard of care for local control in these cases, with 5-year survival rates averaging $60 \%$ to $75 \% .^{2-7}$ The prognosis for patients with recurrent disease in the breast has been correlated with the disease-free interval, the size of the 
recurrence, and the status of the regional lymph nodes. ${ }^{3,4,8-11}$ Nodal staging of the axilla is compromised in patients who have previously undergone a standard level I/II axillary lymph node dissection (ALND). Furthermore, primary lymphatic drainage patterns are likely to have been diverted toward nonaxillary nodal basins as a consequence of the prior ALND, as well as the adjuvant breast radiotherapy. This has been demonstrated to a limited extended in some studies with small sample sizes. $^{12}$ Identification of aberrant lymphatic drainage pathways also helps to explain the pathogenesis of unusual patterns of breast cancer failure, such as contralateral axillary metastases. ${ }^{13}$

Determining the systemic adjuvant therapy needs in locally recurrent breast cancer is challenging. Most local recurrences will be successfully treated by salvage mastectomy alone. A subset of these cases, however, will represent biologically more aggressive breast cancers for which systemic therapy is warranted, but currently available imaging studies are inadequate for identifying these patients. ${ }^{14}$ Identification of regional nodal metastases would be a marker of the high-risk subset ${ }^{9}$; however, surgical staging of the nodes is limited in the context of a conventional completion mastectomy. We hypothesized that lymphatic mapping and sentinel lymph node biopsy in patients with locally recurrent breast cancer would facilitate the ability to surgically stage their regional lymphatic drainage patterns. This study was designed to evaluate the clinicopathologic features, including correlations with lymphatic mapping and lymphoscintigraphy findings, in a series of breast cancer patients treated for local recurrence.

\section{METHODS}

The conduct of this retrospective review was approved by the Institutional Review Board for the University of Michigan. The medical records were reviewed for all breast cancer patients undergoing surgery at the University of Michigan Comprehensive Cancer Center between January 2002 and December 2004 for a diagnosis of local recurrence after prior breast-conservation therapy. Clinical and pathologic characteristics of the initial primary cancer diagnosis and the recurrence were evaluated.

The Surgical Breast Section at the University of Michigan has used lymphatic mapping and sentinel lymph node biopsy in the management of primary breast cancer since 1999, and in 2002 we expanded its application to include patients undergoing comple- tion mastectomy for locally recurrent breast cancer. Because lymphatic mapping would not be considered the standard of care in surgically treating patients with local recurrence from breast cancer, the final decision regarding the use of this approach is left to the discretion of the primary surgeon and the patient. Our mapping procedure has been internally validated for accuracy and is standardized as follows: a subareolar injection of $4 \mathrm{mCi}{ }^{99 m} \mathrm{Tc}$-labeled sulfur colloid (unfiltered) is administered 18 to 24 hours before the scheduled cancer surgery. Preoperative lymphoscintigraphy is performed on the morning of surgery. The primary surgeon injects 3 to $5 \mathrm{~mL}$ of blue dye (Lymphazurin 1\% isosulfan blue dye, U.S. Surgical Corp., Norwalk, CT) into the breast peritumorally and into the dermis overlying the tumor 5 minutes before making the skin incision. The sentinel nodes are identified during surgery by visual inspection, palpation, and use of the handheld gamma detector. The sentinel nodes are evaluated pathologically for evidence of metastases by serial sectioning with hematoxylin and eosin staining.

Lymph nodes retrieved in the surgical specimens of patients with local recurrence were evaluated on the basis of whether lymphatic mapping was performed. Patterns of primary lymphatic drainage (as documented by preoperative lymphoscintigraphy) in the locally recurrent breast cancer cases were compared with patterns observed in our institution for a representative sample of control patients who underwent sentinel node biopsy for primary breast cancer within the same time period. The cases and controls for this study were identified via the University of Michigan Surgical Breast Section procedure records. Comparison of the drainage patterns reported by lymphoscintigraphy was evaluated by $\chi^{2}$ statistical testing.

\section{RESULTS}

Fourteen patients undergoing completion mastectomy for locally recurrent breast cancer were evaluated. The clinicopathologic profile for these patients is shown in Table 1. The median age for these patients at the time of the initial breast cancer diagnosis was 50 years, and the mean time to local recurrence from the date of primary disease detection was 43 months (range, 14-170 months). The median age at the time of local recurrence was 54 years (range, 34-70 years). Ten of these patients (71\%) had lymphatic mapping and sentinel lymph node biopsy performed concomitantly with their completion mastectomy. Lymphatic mapping was performed 
TABLE 1. Clinicopathologic features of the study patient population undergoing treatment for locally recurrent breast cancer

\begin{tabular}{lcccrc}
\hline Patient no. & $\begin{array}{c}\text { Age at primary } \\
\text { CA Dx }(\mathrm{y})\end{array}$ & Stage & Primary CA treatment & $\begin{array}{c}\text { Interval to } \\
\text { local recurrence }(\mathrm{mo})\end{array}$ & $\begin{array}{c}\text { Size of local } \\
\text { recurrence }(\mathrm{cm})\end{array}$ \\
\hline 1 & 42 & T1N1 & L/ALND/XRT & 132 & 1.5 \\
2 & 67 & DCIS & L/XRT & 27 & .3 \\
3 & 33 & T1N1 & L/ALND/XRT & 14 & 3.0 \\
4 & 40 & T2N0 & L/ALND/XRT & 162 & 1.2 \\
5 & 40 & T1N0 & L/ALND/XRT & 68 & .7 \\
6 & 44 & DCIS & L/XRT & 92 & .3 \\
7 & 53 & T1N0 & L/ALND/XRT & 28 & .6 \\
8 & 56 & T1N0 & L/ALND/XRT & 45 & .6 \\
9 & 43 & T1N0 & L/SLNB/XRT & 87 & 1.3 \\
10 & 55 & T1N0 & L/ALND/XRT & 24 & .3 \\
11 & 50 & T2N1 & L/ALND/XRT & 37 & .7 \\
12 & 62 & T1N1 & L/ALND/XRT & 23 & .3 \\
13 & 57 & T2N1 & L/ALND/XRT & 36 & .5 \\
\hline
\end{tabular}

CA, cancer; Dx, diagnosis; L, lumpectomy; ALND, axillary lymph node dissection; XRT, breast radiation; SLNB, sentinel lymph node biopsy.

TABLE 2. Results of lymphatic mapping in nine patients ${ }^{a}$ with locally recurrent breast cancer

\begin{tabular}{|c|c|c|c|c|c|c|}
\hline \multirow[b]{2}{*}{ Patient no. ${ }^{b}$} & \multirow[b]{2}{*}{$\begin{array}{c}\text { Initial } \\
\text { axillary operation }\end{array}$} & \multicolumn{5}{|c|}{ Preoperative lymphoscintigraphy results } \\
\hline & & $\begin{array}{c}\text { Scan not done } \\
\text { (blue dye mapping only) }\end{array}$ & $\begin{array}{l}\text { No drainage on } \\
\text { preoperative scan }\end{array}$ & $\begin{array}{l}\text { Ipsilateral } \\
\text { axilla }\end{array}$ & $\begin{array}{l}\text { Ipsilateral internal } \\
\text { mammary chain }\end{array}$ & $\begin{array}{l}\text { Contralateral } \\
\text { axilla }\end{array}$ \\
\hline 1 & ALND & & & & $\mathrm{X}$ & $\mathrm{x}$ \\
\hline 2 & None & $\mathrm{x}$ & & & & \\
\hline 3 & ALND & & & & $\mathrm{X}$ & $\mathrm{X}$ \\
\hline 4 & ALND & & & & & $\mathrm{X}$ \\
\hline 5 & ALND & & & & & $\mathrm{X}$ \\
\hline 6 & None & & & $\mathrm{x}$ & & \\
\hline 7 & ALND & & & & & $\mathrm{X}$ \\
\hline 8 & ALND & & & & & $\mathrm{x}$ \\
\hline 9 & SLNB & & $\mathrm{X}^{c}$ & & & \\
\hline 10 & ALND & & & & $\mathrm{x}$ & $\mathrm{X}$ \\
\hline
\end{tabular}

ALND, axillary lymph node dissection; SLNB, sentinel lymph node biopsy.

${ }_{b}^{a}$ Four additional patients with local recurrence from this series were managed by completion mastectomy without lymphatic mapping.

${ }^{b}$ Patient numbers correlate with patient numbers from Table 1.

${ }^{c}$ Ipsilateral axillary sentinel lymph node identified by blue dye mapping.

with blue dye as the only mapping agent in one case and with dual agents (blue dye and isotope) in the remaining nine cases. Four patients underwent completion mastectomy alone, with no lymphatic mapping.

Lymphatic mapping and surgical pathology findings from these cases of local recurrence are listed in Table 2. Among the four cases managed by completion mastectomy alone, only one patient had lymph node tissue identified in the surgical specimen, and this incidentally detected lymph node within the axillary tail of the breast was a metastatic lesion. A sentinel node was identified in 9 of the 10 mastectomy cases performed with lymphatic mapping (sentinel identification rate, 90\%), and none of these nodes was metastatic. For the nine cases that included dual-agent lymphatic mapping, radioactive sentinel lymph nodes were identified in eight (89\%). One patient had no radioisotope uptake on preoperative lymphoscintigraphy, and no uptake was detected during surgery, either; this patient did have a single blue-stained sentinel node in the ipsilateral axilla. It is interesting to note that this patient had undergone prior sentinel lymph node biopsy (without completion ALND) at the time of her initial breast cancer operation. The one patient who underwent lymphatic mapping with blue dye only had a single blue node identified in the ipsilateral axilla. The case of sentinel node nonidentification occurred in a patient with lymphoscintigraphy results that revealed contralateral axillary and internal mammary drainage; however, it had been decided before surgery to attempt retrieval only of ipsilateral axillary nodes. 
Eight of the sentinel node specimens identified by isotope mapping were located in the contralateral axillae; three of these patients also had ipsilateral internal mammary chain sentinel nodes. Only one patient had ipsilateral axillary sentinel lymph node location. This patient had been previously treated for ductal carcinoma-in-situ by lumpectomy and breast radiation, but not by ALND.

The lymphatic drainage patterns for these cases of local recurrence were also compared with the patterns observed on preoperative lymphoscintigraphy performed on a sample of 117 patients with primary breast cancer treated within the same time interval as the study population of patients with locally recurrent breast cancer. In the primary breast cancer patients, extra-axillary sentinel nodes were detected in 17 cases (14.5\%): supraclavicular in 12; internal mammary in 4 , and internal mammary as well as supraclavicular in 1. Concomitant ipsilateral axillary drainage was detected in all 12 of these cases. The difference in the rate of nonipsilateral axillary drainage between the primary breast cancer and locally recurrent breast cancers, as documented by lymphoscintigraphy, was statistically significant $(14.5 \%$ vs. $66.7 \% ; P=.001)$.

The median number of lymph nodes retrieved for pathology evaluation in the local recurrence cases managed by mastectomy only was 1 (range, $0-1$ ), versus 3 (range, 1-6) in the cases in which lymphatic mapping and sentinel node dissection were performed. The median number of sentinel nodes retrieved among the patients who underwent sentinel node dissection for early-stage primary breast cancer was also 3 .

\section{DISCUSSION}

Advances in breast cancer management, including an expanded armamentarium of hormonally active agents, chemotherapy, and novel targeted therapies, have resulted in improved disease-related outcomes. Furthermore, several trends in cancer surveillance and treatment are likely to improve eligibility for breast conservation therapy ${ }^{15}$ :

1. Increasing incidence rates of breast cancer among young women.

2. Earlier detection of breast cancer via screening mammography, with more tumors detected at sizes that are amenable to lumpectomy.

3. Expanded applications for neoadjuvant chemotherapy, with tumor downstaging and increased breast-conservation therapy eligibility.

As the subset of conservatively treated breast cancer patients enlarges, in conjunction with prolonged survival from the disease, we are likely to see an increased number of women with either true inbreast recurrences or new primary tumors in the previously treated breast. The survival equivalence of breast-conserving approaches compared with mastectomy for stage-matched patients has been definitively documented in multiple phase III clinical trials. ${ }^{15-18}$ Although it has been definitively documented by multiple phase III clinical trials that overall survival from breast cancer is equivalent for stage-matched patients regardless of the choice for lumpectomy versus mastectomy, it has also been demonstrated that the development of a local recurrence is a marker of inherently more aggressive primary tumor biology. ${ }^{8}$ A local recurrence in the breast is associated with a 3 to 5 times greater risk for experiencing distant failure. ${ }^{8}$

Salvage mastectomy is currently the standard-ofcare management for patients who develop ipsilateral cancerous lesions after previous treatment by lumpectomy and breast radiation, and approximately two thirds of these patients will have prolonged diseasefree survival after the completion mastectomy. Collectively, these patterns suggest that a subset of in-breast recurrences are indicators of high-risk disease for which systemic adjuvant therapy would be warranted; another subset (the majority) are cases that will be adequately controlled by more extensive local therapy alone. Identifying these disparate subsets at the time of local recurrence has been an ongoing challenge to clinicians involved in the multidisciplinary management of breast cancer since the breast-conservation trials were initially completed. Table 3 summarizes the results of selected studies that have attempted to identify prognostic features associated with outcome after completion mastectomy for local recurrence. As shown, there are no consistent characteristics to determine when systemic therapy is indicated for a local recurrence. The infrequency of nodal metastases in our series of local recurrences (1 in 14) supports the concept that only a minority of in-breast failures represent biologically aggressive disease.

The disease-free interval, the size of an invasive local recurrence, and the presence of nodal metastases at the time of recurrence have been correlated with the need for systemic therapy to address distant micrometastases. As with primary breast cancer, the presence of regional nodal disease is an excellent 
TABLE 3. Selected studies of prognostic features in patients with locally recurrent breast cancer

\begin{tabular}{lccl}
\hline Study & No. of local recurrences & 5-y overall survival $(\%)$ & Adverse prognostic features $^{a}$ \\
\hline Kurtz, $1989^{7}$ & 178 & 69 & DFI $<5 \mathrm{y}$ \\
Stotter, 1989 & 55 & 63 & Local recurrence $>5 \mathrm{~cm}$; unresectable nodes at time of recurrence \\
Cajucom, $1993^{4}$ & 25 & 65 & Local recurrence $>2 \mathrm{~cm} ; \geq 4$ metastatic nodes at time of recurrence \\
Voogd, 1999 & 266 & 61 & Local recurrence $>1 \mathrm{~cm}$; nodal status of the primary tumor \\
Dalberg, 2003 & 68 & DFI $<3$ y; nodal status of the primary tumor \\
\hline
\end{tabular}

DFI, disease-free interval (time to local recurrence).

${ }^{a}$ Features associated with poor outcome after completion mastectomy.
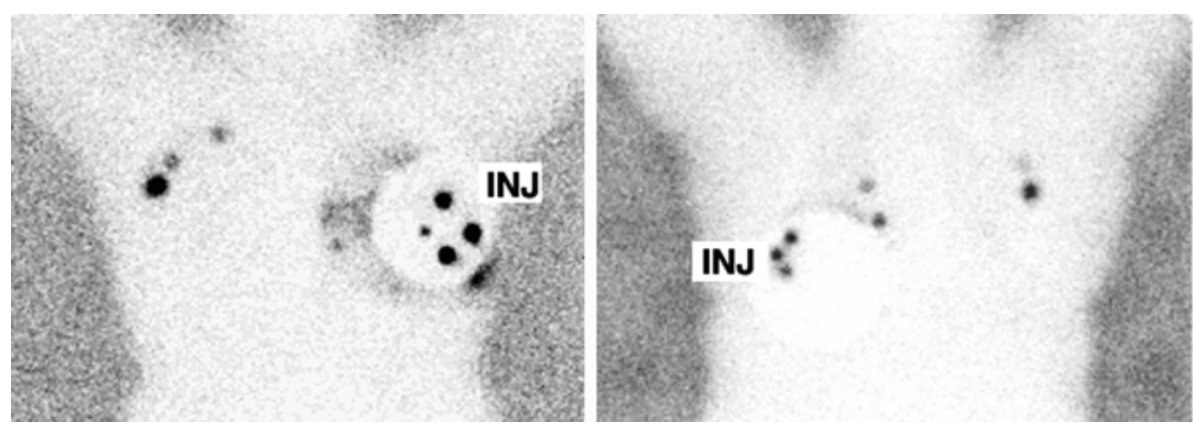

FIG. 1. Preoperative lymphoscintigraphy in a patient with local recurrence undergoing completion mastectomy, revealing drainage to the contralateral axilla and ipsilateral internal mammary nodal chains. INJ, periareolar injection sites. surrogate marker for potential micrometastases in distant organs. Most invasive breast cancer patients treated $>5$ years ago, however, will have already undergone an ipsilateral ALND. This results in an anatomically altered axillary fat pad for resection in cases in which completion mastectomy is required for management of a local recurrence. It is likely that the primary lymphatic drainage of the breast will have been rerouted as a consequence of the previous axillary surgery, and breast radiation may alter intramammary lymphatic patterns as well.

The conventional salvage mastectomy performed for locally recurrent breast cancer is therefore inadequate for obtaining information regarding the regional extent of disease. Foci of occult metastasis in the ipsilateral axilla will be more difficult to identify, and lymphatic drainage to the alternative nodal basins will be overlooked completely. The technology of lymphatic mapping and sentinel lymph node biopsy for breast cancer offers a unique opportunity to explore and restage the patient who experiences a local recurrence from breast cancer. Dominant lymphatic drainage to extra-axillary nodal basins, such as the internal mammary chain, the supraclavicular fossa, and even the contralateral axilla, is identified by lymphatic mapping and preoperative lymphoscintigraphy in approximately $10 \%$ of patients who undergo surgical treatment for primary breast cancer.

This study confirmed our a priori hypothesis that primary lymphatic drainage patterns in the breast are different after an ALND with or without breast irra- diation. Lymphatic mapping with radioisotope injection and lymphoscintigraphy in our subset of patients who underwent mastectomy for locally recurrent breast cancer revealed drainage to extra-axillary sentinel lymph nodes in nearly all cases. Because regional metastatic spread of disease will similarly be affected, this information can direct the surgical staging plan, and systemic therapy recommendations can take into account the presence versus the absence of disease in any aberrantly located sentinel nodes. Figure $1 \mathrm{dem}-$ onstrates the lymphoscintigraphy results in a patient who underwent completion mastectomy after prior ALND and had primary lymphatic drainage detected in the internal mammary and contralateral axilla. We included four cases of salvage mastectomy alone (without lymphatic mapping) performed for local recurrence during the same time frame as our study patients, and this sample demonstrated the typical scenario of few or no lymph nodes retrieved with this "conventional" approach. Only three of the patients in our series received chemotherapy for their local recurrence - the one patient with nodal failure, another patient with a 5-cm invasive recurrence, and the patient whose mapping was unsuccessful in identifying any sentinel lymph nodes. Had any of the other patients been found to harbor metastases in their aberrantly located nodes, it is likely that they would have received systemic therapy as well.

Patients with local recurrence after prior lumpectomy and limited axillary surgery (e.g., sentinel lymph node biopsy) are also likely to benefit from 
lymphatic mapping and sentinel node biopsy at the time of their in-breast relapse. There are few data reported thus far on the feasibility of repeat mapping procedures in breast cancer patients. The surgical breast group at Memorial Sloan-Kettering Cancer Institute reported on results from "reoperative" sentinel lymph node biopsy in a series of 32 patients who had undergone prior axillary surgery for both benign and malignant conditions, and they had an identification rate of $75 \% .{ }^{19}$ Our group has looked at the feasibility of sequential sentinel node biopsy procedures in neoadjuvant chemotherapy patients before and after the induction systemic therapy, with identification rates of $92 \% .^{20}$ This study included only one patient whose initial breast cancer had been staged by prior axillary sentinel lymph node biopsy. This patient had another axillary sentinel node detected by blue dye mapping at the time of her mastectomy for local recurrence. Even in cases of local recurrence after breast conservation with no prior axillary operation, lymphatic mapping may improve staging of the relapse, because the breast radiation may have altered the primary lymphatic drainage patterns.

In summary, our study demonstrates that lymphatic mapping for locally recurrent breast cancer is feasible. Lymphatic mapping performed with radioisotope and lymphoscintigraphy is likely to identify sentinel nodes located in the contralateral axilla or the internal mammary chain. Blue dye in addition to isotope mapping increases the sentinel node identification rate. We believe that the nodal staging of patients who experience a local recurrence after breast-conservation therapy can potentially contribute meaningful information regarding the underlying tumor biology and that these findings can be incorporated into recommendations for systemic therapy. Our findings require validation in other breast cancer datasets so that their prognostic value can be defined.

\section{REFERENCES}

1. Ries L, Eisner M, Kosary CL, et al. SEER Cancer Statistics Review, 1973-1999. Vol. 2003. Bethesda, MD: National Cancer Institute, 2002.

2. Stotter AT, McNeese MD, Ames FC, Oswald MJ, Ellerbroek NA. Predicting the rate and extent of locoregional failure after breast conservation therapy for early breast cancer. Cancer 1989; 64:2217-25.
3. Voogd AC, Tienhoven G, Peterse HL, et al. Local recurrence after breast conservation therapy for early stage breast carcinoma: detection, treatment, and outcome in 266 patients. Dutch Study Group on Local Recurrence after Breast Conservation (BORST). Cancer 1999; 85:437-46.

4. Cajucom CC, Tsangaris TN, Nemoto T, Driscoll D, Penetrante RB, Holyoke ED. Results of salvage mastectomy for local recurrence after breast-conserving surgery without radiation therapy. Cancer 1993; 71:1774-9.

5. Haffty BG, Carter D, Flynn SD, et al. Local recurrence versus new primary: clinical analysis of 82 breast relapses and potential applications for genetic fingerprinting. Int $J$ Radiat Oncol Biol Phys 1993; 27:575-83.

6. Kurtz JM, Amalric R, Brandone H, et al. Local recurrence after breast-conserving surgery and radiotherapy. Frequency, time course, and prognosis. Cancer 1989; 63:1912-7.

7. Kurtz JM, Amalric R, Brandone H, Ayme Y, Spitalier JM. Local recurrence after breast-conserving surgery and radiotherapy. Helv Chir Acta 1989; 55:837-42.

8. Lannin DR, Haffty BG. End results of salvage therapy after failure of breast-conservation surgery. Oncology (Huntingt) 2004; 18:272-9; discussion 280-2, 285-6, 292.

9. Osborne MP, Simmons RM. Salvage surgery for recurrence after breast conservation. World J Surg 1994; 18:93-7.

10. Krauss DJ, Kestin LL, Mitchell C, Martinez AA, Vicini FA. Changes in temporal patterns of local failure after breastconserving therapy and their prognostic implications. Int $J$ Radiat Oncol Biol Phys 2004; 60:731-40.

11. Dalberg K, Liedberg A, Johansson U, Rutqvist LE. Uncontrolled local disease after salvage treatment for ipsilateral breast tumour recurrence. Eur J Surg Oncol 2003; 29:143-54.

12. Sood A, Youssef IM, Heiba SI, et al. Alternative lymphatic pathway after previous axillary node dissection in recurrent/ primary breast cancer. Clin Nucl Med 2004; 29:698-702.

13. Daoud J, Meziou M, Kharrat M, Sellami D, Boudawara T, Frikha M. Contralateral axillary lymph node metastasis of cancer of the breast (in French). Bull Cancer 1998; 85:713-5.

14. van Oost FJ, van der Hoeven JJ, Hoekstra OS, Voogd AC, Coebergh JW, van de Poll-Franse LV. Staging in patients with locoregionally recurrent breast cancer: current practice and prospects for positron emission tomography. Eur $J$ Cancer 2004; 40:1545-53.

15. Newman LA, Washington TA. New trends in breast conservation therapy. Surg Clin North Am 2003; 83:841-83.

16. Fisher B, Anderson S, Bryant J, et al. Twenty-year follow-up of a randomized trial comparing total mastectomy, lumpectomy, and lumpectomy plus irradiation for the treatment of invasive breast cancer. $N$ Engl J Med 2002; 347:1233-41.

17. Poggi MM, Danforth DN, Sciuto LC, et al. Eighteen-year results in the treatment of early breast carcinoma with mastectomy versus breast conservation therapy: the National Cancer Institute Randomized Trial. Cancer 2003; 98:697-702.

18. Veronesi U, Cascinelli N, Mariani L, et al. Twenty-year followup of a randomized study comparing breast-conserving surgery with radical mastectomy for early breast cancer. $N$ Engl $J$ Med 2002; 347:1227-32.

19. Port ER, Fey J, Gemignani ML, et al. Reoperative sentinel lymph node biopsy: a new option for patients with primary or locally recurrent breast carcinoma. J Am Coll Surg 2002; 195: $167-72$.

20. Khan A, Sabel M, Nees A, et al. Comprehensive axillary evaluation in neoadjuvant chemotherapy patients with ultrasound and sentinel lymph node biopsy. Ann Surg Oncol 2005; 12:697-704 